\title{
Ein Altersheim als Zentrum einer Region
}

\author{
Hoch oben im Onsernonetal steht seit 1989 in Russo das Centro Sociale Onsernonese. \\ Über die Kernfunktion eines Alters- und Pflegeheim hinaus ist es ein wichtiger sozia- \\ ler Treffpunkt sowie der grösste Arbeitgeber der Region. Das im «Centro» unterge- \\ brachte, durch den Arzt Beppe Savary-Borioli geleitete, medizinische Ambulatorium \\ stellt zudem die medizinische Versorgung des gesamten Tales sicher. Massgeblich \\ am Erfolg des Projekts beteiligt ist die Schweizer Patenschaft für Berggemeinden.
}

Rob Neuhaus

Korrespondenz: Rob Neuhaus

Texte - Kommunikation Hauptgasse 35 CH-5466 Kaiserstuh
Eine total verrückte Idee war das aus heutiger Sicht. Aber aus einer Utopie, aus einem Traum wurde Realität. Beppe Savary-Borioli, Arzt im Onsernonetal, und Alfredo Gamboni, pensionierter Lehrer, blicken auf die lange Geschichte des Centro Sociale Onsernonese CSO, des Alters- und Pflegeheims für die Talschaft, zurück. Sie haben diese Geschichte massgeblich geprägt. Gamboni hatte als langjähriger Präsident der Trägervereinigung über 1200 Sitzungen geleitet, bis das Centro 1989 seinen Betrieb aufnehmen konnte. Zehn Ordner mit Protokollen zeugen von dieser Arbeit. Savary-Borioli sorgte mit seiner praktischen Art immer dafür, dass ein Centro geplant und gebaut wurde, das möglichst vielen Bedürfnissen entsprach, und zusammen haben sie das Projekt über viele Hürden - bürokratische und finanzielle - gebracht.

\section{Das Centro, ein Multifunktionsbetrieb}

Entstanden ist ein Ort, der das Tal prägt - und am Leben erhält. Denn das Centro ist nicht einfach ein

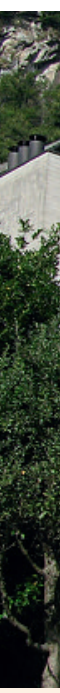

\section{7}

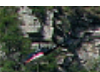

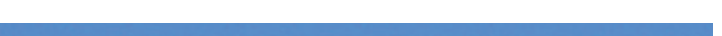
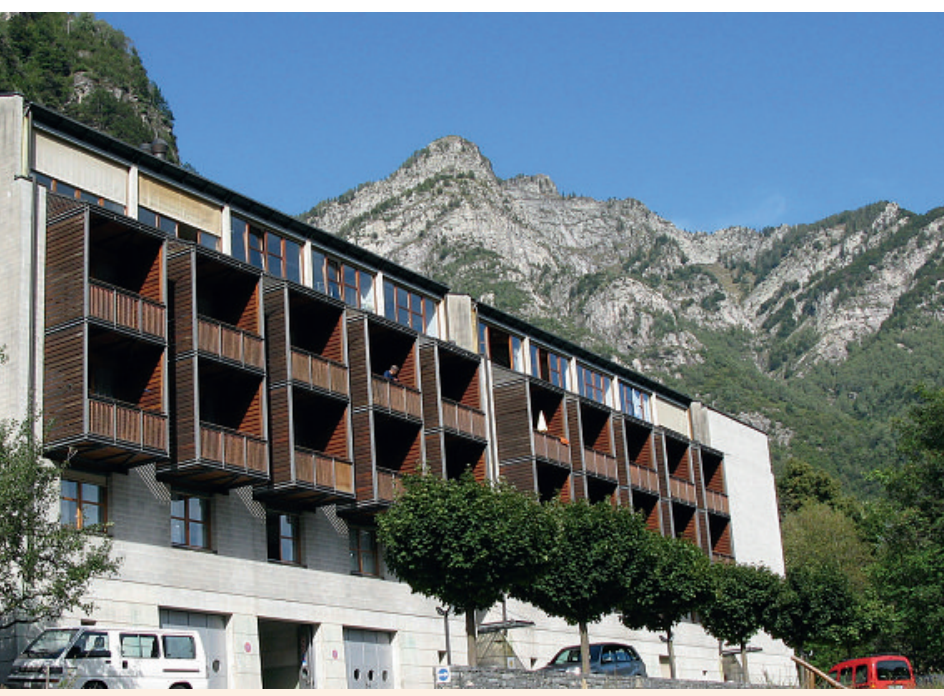

Das Centro: Alle Zimmer haben einen Holzbalkon im historischen Baustil. Sie sind so eingerichtet, dass Betten und Infrastruktur auch für Pflegefälle aufgerüstet werden können.
Altersheim, das den Menschen ein Leben in Würde erlaubt, sondern ein vitaler Treffpunkt mitten im Dorf Russo. Etwa vierzig Männer und Frauen wohnen hier in Zimmern, die zwar einfach ausgestattet sind, es aber erlauben, dass die Bewohner Möbel, Bilder, Einrichtungsgegenstände mitbringen, die ihnen ans Herz gewachsen sind. Alle Zimmer haben einen Balkon, ganz im Baustil des Tales, mit Blick auf die gegenüberliegenden üppig grünen Hänge des Pizzo Ruscada. Die früheren Generationen trockneten auf den Holzvorbauten Stroh. Die Verarbeitung des Strohs zu Hüten und anderen Produkten brachte vielen Menschen eine willkommene Erwerbstätigkeit und jenen, die im Ausland damit handelten, sogar einen gewissen Reichtum. Zahlreiche barocke Palazzi und verzierte Gräber sind Zeugen davon.

Vor dem Centro, auf der kleinen Piazza und in der Bar, die ebenfalls zum Betrieb gehört, treffen sich Leute aus dem Dorf zum Kaffee und zum Plaudern. Nebenan befindet sich die gedeckte Bocciabahn - natürlich

\section{Centro Sociale Onsernonese}

Das Centro Sociale Onsernonese CSO betreut zwei Alters- und Pflegeheime in Russo und Loco. Das Patriziato Generale d'Onsernone (Ortsbürgerschaft) kam 1978 zum Schluss, dass das bestehende private Altersheim in Loco, das von den Schwestern der Heiligen Familie aus dem Kloster in Castelletto del Garda in Italien geführt wurde, für die Senioren des Tals nicht ausreichte. 1989 wurde der Neubau in Russo eröffnet, 2003 konnte das Haus in Loco dazugekauft und umgebaut werden. Das Centro beherbergt rund 60 Pensionäre (40 in Russo, 20 in Loco) und beschäftigt gut 100 Mitarbeitende (60 Vollzeitstellen).

Damit dieser multifunktionale Betrieb wirken kann, ist er auf Unterstützung angewiesen - mit der Schweizer Patenschaft für Berggemeinden ist im Verlauf der Jahre eine gut funktionierende und fruchtbare Beziehung entstanden. 
homologiert, damit hier auch offizielle Turniere stattfinden können. Und Punkt zwölf Uhr stürmt eine Schar Kinder in den Esssaal - denn die Köche des Centro kochen nicht nur für die Gäste und das Personal, sondern auch für die Schule. Im Untergeschoss ist das medizinische Ambulatorium eingerichtet, welches die ärztliche Versorgung nicht nur für das Altersund Pflegeheim, sondern auch für das ganze Tal gewährleistet. Und schliesslich arbeiten hier Leute: Rund 100 Personen, vorwiegend aus den Dörfern des Tales, finden hier ein Auskommen oder einen Zusatzverdienst. Als 2003 noch der Sitz in Loco, der von den Schwestern der Heiligen Familie aufgegeben worden war, übernommen werden konnte, war der Betrieb komplett. So ist das Centro tatsächlich ein Zentrum sozial, medizinisch, geografisch, wirtschaftlich und alle Generationen übergreifend.

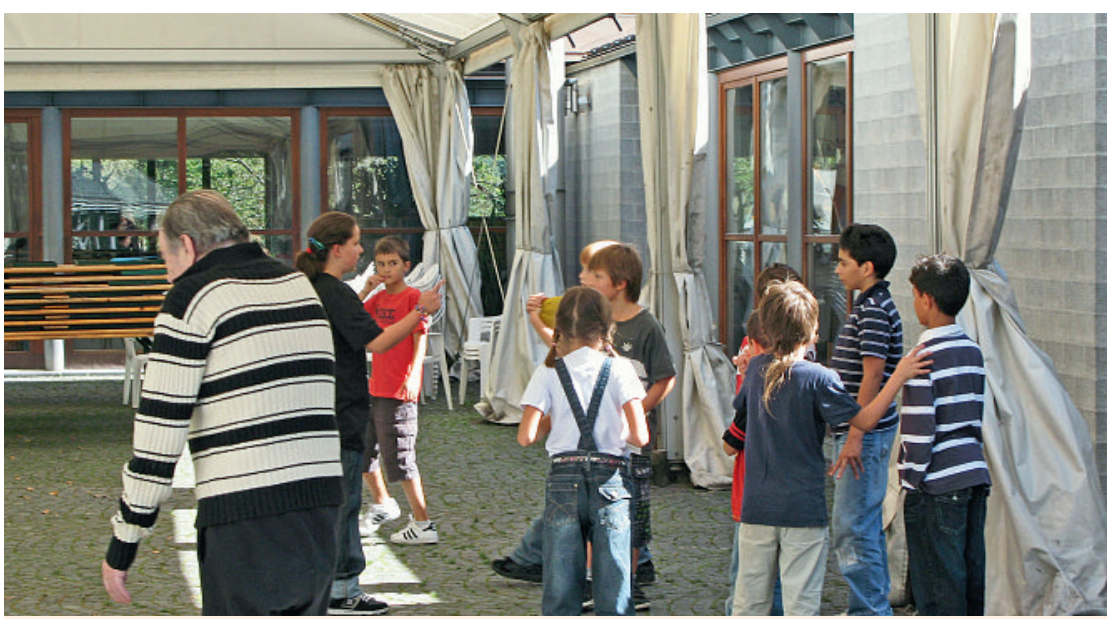

Das Centro ist auch Mittagstisch der Schule. Über Mittag kommt Leben ins Haus - die Jungen treffen die Alten.

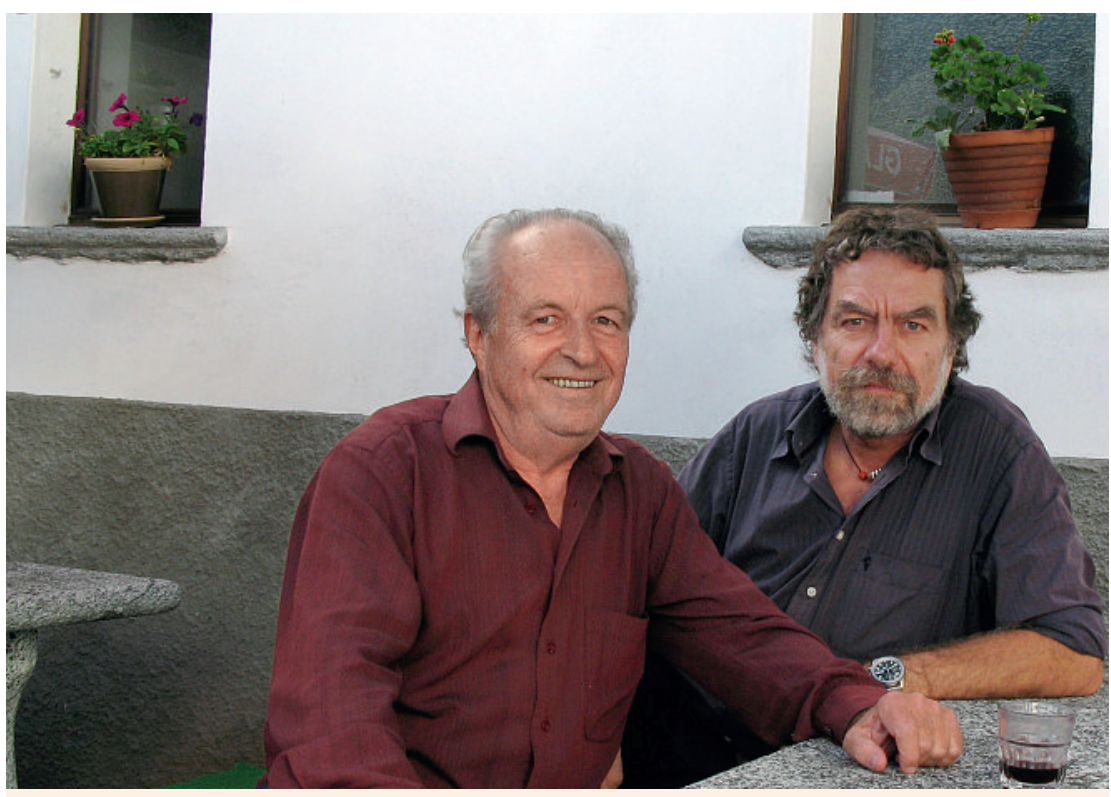

1200 Sitzungen, bis nur schon der Betrieb des CSO aufgenommen werden konnte: Alfredo Gamboni (links), Gründungspräsident des CSO, und Beppe Savary-Borioli (rechts).

\section{Ein Altersheim als Jungbrunnen}

Für die Gäste ist der Aufenthalt im Altersheim der letzte Lebensabschnitt. Für das ganze Tal hingegen bedeutet das Centro den Anschluss ans Leben. Mit den rund 100 Mitarbeitenden in Russo und Loco, das entspricht gut 60 Vollzeitstellen, ist das Centro der grösste Arbeitgeber der Region. Das ermöglicht nicht nur zahlreichen Familien, im Tal zu bleiben, sondern ist auch ein Anreiz, ins Tal zu ziehen, nachdem während Jahrzehnten Menschen das Tal verliessen. Heute gibt es genug Familien mit Kindern, so dass die Existenz der Schule bis zur Oberstufe gesichert ist. In der Küche des Centro werden lokale, biologische Produkte zubereitet, und die fünf Lehrlinge, die bisher hier ihre Ausbildung abschlossen, arbeiten heute in renommierten Hotels rund um die Welt. Vereine, Restaurants und Läden können überleben und ihren Beitrag zum Leben leisten. «Wichtig für unser Tal ist nicht nur, dass die Leute hier bleiben oder hierher ziehen können», meint Savary-Borioli, «sondern, dass sie hier auch Schule und Ausbildung finden. Das erst gibt eine solide Grundlage.»

\section{Hohe Hürden im tiefen Tal}

«Ohne die Patenschaft für Berggemeinden gäbe es uns nicht,» sagt Beppe Savary-Borioli. Er hat alle Schwierigkeiten kennengelernt, die sich einem Projekt solchen Ausmasses im finanziell schwachen Tal in den Weg stellen. Und doch hat er nie aufgehört, die besten Ideen verwirklichen zu wollen. Dazu gehört nicht nur die Holzschnitzelheizung, die das Holz aus dem Tal verwertet. «Vor zwanzig Jahren war das noch einzigartig,» schmunzelt er. Dazu gehört auch die Architektur, die sich am lokalen Baustil orientiert und das Licht des Tals in alle Räume lässt, in denen gelebt wird. Und schliesslich gehören dazu auch wichtige Details wie drei offene Feuerstellen, verteilt auf verschiedene Stockwerke. Warum es denn drei Feuerstellen sein müssten, eine sei doch genug, war die Reaktion der kantonalen und eidgenössischen Behörden, die die Finanzierung gutheissen mussten. Die Menschen müssten sich eben wie zu Hause fühlen können, antwortete Savary-Borioli. Und er hatte recht. Im Herbst sitzen die Leute vor den Feuern zusammen, plaudern und rösten Kastanien, wärmen sich Hand und Herz am offenen Feuer.

\section{Ein Notarzt für alle Fälle}

Beppe Savary-Borioli leitete das medizinische Ambulatorium seit Beginn, lange Jahre zusammen mit seinem

\section{Medizinisches Ambulatorium}

Im Centro Sociale Onsernonense CSO befindet sich das medizinische Ambulatorium. Es ist organisatorisch und administrativ integriert. Der medizinische Leiter ist Dr. Beppe Savary-Borioli. Das Ambulatorium stellt die medizinische Versorgung des Centro und der ganzen Talschaft rund um die Uhr sicher. 
Bruder und seit dessen Tod 2007 mit Stefano Mozzettini. Savary-Borioli ist zu 50 Prozent angestellt, daneben ist er der Leiter des Rettungsdienstes Locarnese e valli SALVA. Beide haben langjährige Erfahrung in Bergrettung und Notfallmedizin. Mit ihrem Team von Assistenten, Stagiaires und Lernenden sind sie rund um die Uhr im Einsatz. Savary-Borioli hat, bis auf einige Jahre, als er dem Wunsch der Familie folgend in Locarno wohnte und in Lugano arbeitete, hier im Onsernonetal gearbeitet. Obwohl er ein Zugewanderter ist ein «matlosa» oder Heimatloser, wie die Einheimischen sagen - kennt er längstens jedes Haus, von zuunterst bis zuoberst im Tal mit allen seinen Bewohnern. Wenn er mit seinem Auto, einer «fahrenden Intensivstation», durch die Dörfer fährt, kennt er nicht nur jede Kurve und jeden Engpass, sondern grüsst auch jeden Passanten am Strassenrand. Die Krankengeschichten dieser Menschen führt er in seinem Gedächtnis gleich mit sich.

\section{Telemedizin im Randgebiet}

Das Einsatzgebiet des Rettungsdienstes Locarnese e valli ist die grösste Region des Tessins und ist mit der zweithöchsten Anzahl medizinischer Einsätze im Kanton konfrontiert. Relativ viele Unfälle bei Sport, Jagd und Verkehr tragen zu dieser hohen Rate bei. Das weitläufige Gebiet und die kurvenreiche und enge Strasse des Onsernonetals - rund dreissig Kilometer von Locarno bis zuhinterst nach Spruga - hat lange Fahrzeiten zur Folge. Bei gutem Wetter können Patienten schnell ins Spital von Locarno oder Lugano geflogen werden, auf das Wetter ist aber auch in der sogenannten Sonnenstube kein Verlass. So hat Savary sich schon vor einer Weile auf die elektronische Übermittlung medizinischer Patientendaten spezialisiert. «In unserer Region ist es wichtig, dass wir mittels moderner Technologie die Distanzen überwinden können. So kann ich jederzeit nicht nur auf die Patientendaten des Centro, sondern auch anderer Spitäler zugreifen», so SavaryBorioli. Und nicht ohne Stolz sagt er: «In der Telemedizin sind wir, als Tessiner Ambulanzverband, führend.»

Dringend war denn auch der Ersatz der über zwanzigjährigen Röntgenanlage. Der Vorteil liegt auf der Hand: kein umweltbelastendes Entwicklungslabor, keine Analognegative, die irgendwann verloren gehen, sondern digitale Bilder, die sofort auch an Spezialisten gesandt werden können, um über Massnahmen zu entscheiden. Die Finanzierung für diese Pionierleistung stellte für das Centro allerdings wieder eine besondere Aufgabe dar. Denn die Viertelmillion Franken, die die Röntgenanlage kostete sowie weitere wichtige Neuanschaffungen liessen sich unmöglich aus dem Betrieb finanzieren. Dank dem guten Kontakt zur Patenschaft für Berggemeinden fand sich aber auch hier wieder eine Lösung.

\section{Onsernonetal}

Das Onsernonetal liegt zwischen Centovalli und dem Maggiatal. Das wilde, tief eingeschnittene Tal beginnt oberhalb Spruga und endet bei Intragna. Ab dem sechzehnten Jahrhundert bestimmte die Strohverarbeitung das Leben im Tal. Zahlreiche Familien verkauften die Strohprodukte, vor allem Hüte, auf den Märkten Europas und kamen dadurch zu Reichtum. Zurück im Tal, liessen sie sich Palazzi bauen, welche heute noch die Dorfbilder prägen. Inklusive dem Seitental Vergeletto wohnen knapp tausend Menschen in der Region. Von den gut 160 Arbeitsplätzen im Onsernonetal sind 120 im Dienstleistungssektor.

\section{Schweizer Patenschaft für Berggemeinden} Aus dem Solidaritätsgedanken zwischen Berg und Tal entstand 1940 die Schweizer Patenschaft für Berggemeinden mit dem Ziel, durch projektbezogene Hilfe das Gefälle zwischen wohlhabenden und wirtschaftlich benachteiligten Regionen abzubauen. Gründer waren das Ärztepaar Olga und Paul Cattani und weitere Initianten. Heute bearbeitet die Patenschaft jährlich rund 400 Gesuche von Gemeinden und öffentlich-rechtlichen Körperschaften. In enger Zusammenarbeit mit den zuständigen Kantonen, Gemeinden und zielverwandten Organisationen klärt die Patenschaft die Finanzlage und die Unterstützungswürdigkeit der Projekte $a b$ und sucht und vermittelt finanzstarke Gemeinden wie auch private Gönner und Organisationen. 2009 konnte die Patenschaft gut 20 Millionen Franken vermitteln. Die Organisation ist ein Verein; die Geschäftsstelle befindet sich in Zürich. 